\title{
Occurrence of Acute Coronary Syndrome, Pulmonary Thromboembolism and Cerebrovascular Event in COVID-19
}

\author{
yaser jenab ${ }^{1}$, nima rezaei ${ }^{2}$, mohammadreza naderian ${ }^{1}$, behnam hedayat ${ }^{1}$, shahpour \\ shirani $^{1}$, and kaveh hosseini ${ }^{1}$ \\ ${ }^{1}$ Tehran Heart Center \\ ${ }^{2}$ Tehran University of Medical Sciences
}

May 14, 2020

\begin{abstract}
COVID-19 causes significant hypoxia along with an inflammatory milieu, which may lead to secondary myocardial infarction and also atherosclerotic plaque rupture. Here we present a case of COVID-19 presented with ACS followed by PTE and CVA.
\end{abstract}

\section{Introduction}

In December 2019, the Corona virus emerged in the Huanan Seafood Market, Wuhan, China, and rapidly became a worldwide problem[1]. Now, coronavirus disease (COVID-19) pandemic is considered to be a real crisis[2]. About $80 \%$ of patients with COVID-19 seem to mild or even no symptoms[3], however some may experience severe illness.

Atrial and venous thrombotic events have been reported in COVID-19 patients[4]. Hypoxia, severe inflammatory response, and baseline traditional risk factors predispose patients to thrombotic events. Cytokine storm in COVID-19 is the plausible mechanism for thrombotic events. Acute coronary syndrome, pulmonary thromboembolism (PTE), and cerebrovascular accidents (CVA) all may happen in COVID-19 patients due to inflammatory milieu and hemostatic changes.

Here, we report a 70-year old COVID-19 positive patient, presenting with acute coronary syndrome which has been complicated by acute PTE and CVA.

\section{Case Presentation}

A 70-year-old woman was referred to the Emergency Department of Tehran Heart Center with chief complaints of typical chest pain and cold perspiration. Her symptoms had started 4 days earlier, but fever was added to her presentation on the day of admission. Unfortunately, due to the panic among the general population regarding the transmission of COVID-19, the patient ignored her symptoms at the beginning; she came just after increasing in the severity and frequency of her chest pain.

Physical examination revealed a body temperature of $38.6^{\circ} \mathrm{C}$, a respiratory rate of 20 per minute, a blood pressure of $100 / 65 \mathrm{~mm} \mathrm{Hg}$, a heart rate of $110 \mathrm{bpm}$, and an $\mathrm{O}_{2}$ saturation level of $89 \%$. The patient was ill and appeared toxic; nonetheless, she had a normal consciousness level and was well-oriented.

She has previous history of diabetes mellitus, hypertension, and dyslipidemia. Additionally, she had undergone coronary artery bypass graft surgery two years ago in this hospital. 
Electrocardiography on admission illustrated sinus tachycardia, generalized ST- depressions, and STelevations in aVR and $\mathrm{V}_{1}$; all in favor of a left-main pattern or multi-vessel ischemia. She subsequently underwent echocardiography, which demonstrated a left ventricular ejection fraction (LVEF) of $45 \%$, mild right ventricular (RV) dysfunction, and moderate mitral and tricuspid regurgitation. The patient's echocardiogram obtained a year before was almost the same, except for mild tricuspid regurgitation.

An interventional cardiologist was consulted immediately. Given the patient's age, fever, and suspicion of COVID-19, a decision was made to administer full anticoagulant and anti-ischemic therapy and obtain a chest computed tomography (CT) scan. Her chest pain subsided and she became stable; nevertheless, she remained febrile. The patient underwent another chest CT scan (Fig. 1), which showed mild-to-moderate pleural effusion. Based on her symptoms, a real-time reverse transcription-polymerase chain reaction (rRTPCR) test for COVID-19 was requested, and hydroxychloroquine was initiated based on infectious disease consultation.

Ten hours later (the next day), the patient experienced an increase in her chest pain. She was transferred to the catheterization laboratory. The anastomosis of the left internal mammary artery to the left anterior descending artery was opened, but the anastomosis of the saphenous vein graft to the left circumflex artery $\left(\mathrm{LC}_{\mathrm{X}}\right)$ was occluded and the $\mathrm{LC}_{\mathrm{X}}$ was $90-99 \%$ stenotic. Percutaneous coronary intervention (PCI) was performed on the $\mathrm{LC}_{\mathrm{X}}$. Post procedurally, the patient experienced a relief in her chest pain and became stable, but her fever persisted and her $\mathrm{O}_{2}$ saturation level was 89-90\%. Dry coughs became more prominent. The second spiral chest CT scan was compatible with COVID-19, and the rRT-PCR result was also positive for COVID-19. Accordingly, lopinavir/ritonavir (KALETRA) was added to her drug regimen, and $\mathrm{O}_{2}$ therapy under the supervision of an anesthesiologist was continued. Her laboratory tests revealed a white blood cell count of 10,700, a lymphocyte count of 1070, a C-reactive protein level of $2.5(>0.5 \mathrm{mg} / \mathrm{dL})$, a creatinine level of $2.1 \mathrm{mg} / \mathrm{dL}$, a brain natriuretic peptide level of $530 \mathrm{pg} / \mathrm{mL}$, and a troponin level of $269 \mathrm{ng} / \mathrm{L}$. Her blood culture was negative.

After 10 days of treatment with anti-corona drugs, the patient's symptoms worsened and her $\mathrm{O}_{2}$ saturation level dropped to $70 \%$. Chest CT scan illustrated diffuse ground-glass pattern in the parenchyma. This chest $\mathrm{CT}$ scan was discernibly worse than the previous one.

In the meantime, the patient's persistent post-PCI tachycardia, hypoxia, and moderate immobility urged us to investigate other possible causes of hypoxia such as tamponade, mechanical complications of myocardial infarction, and acute pulmonary thromboembolism (PTE). In addition, she experienced weakness and paresthesia of the right arm. Bedside echocardiography showed an LVEF of 40-45\%, moderate RV dysfunction, moderate mitral regurgitation, and severe tricuspid regurgitation. Pulmonary CT angiography demonstrated bilateral, lobar PTE with an RV/LV of about 0.93 (Fig. 2). As the patient had been on prophylactic heparin since PCI, the therapeutic dosage was resumed. Unfortunately, in Brain CT a hypo-dense area in left parietooccipital area and also semiovale area in favor of watershed infarct was noted (Fig. 2).

Fortunately, she survived and was discharged from the hospital in an acceptable condition and with stable vital signs. The diagnosis of PTE and initiation of therapeutic heparin clearly improved her condition. Stroke rehabilitation was started from hospital and requested in outpatient visits.

\section{DISCUSSION}

The patient was an old woman with multi risk factors suffering from acute coronary syndrome, COVID 19, PTE and acute CVA. Recent reports indicated that older patients with cardiovascular disease constitute the highest-risk group. This high-risk group is likely to have coagulopathy; hence, an interim guideline was issued by the International Society on Thrombosis and Hemostasis, suggesting low-dose prophylactic heparin for the management of coagulopathy[5]. Venous thromboembolism threatens patients with COVID-19 and adds the risk of acute respiratory distress syndrome. Venous thromboembolism seems to remain underdiagnosed in COVID-19 patients. These patients have pulmonary hypertension and right ventricular dysfunction and small acute PTE may deteriorate the condition. 
Pulmonary thromboembolism occurred in 20\% of COVID-19 ICU patients during 1 month descriptive study by Poissy $\mathrm{J}$ et al ; twice the number in the same duration in 2019. Of note, the proportion of PTE in COVID-19 was estimated to be twice the number in influenza outbreak[6].

Large vessel stroke has also been discussed in association with COVID-19 in another study by Oxley et al[7]. In the recent study by Yanan Liet al, a single center observational study was performed on 221 COVID-19 patients. In their registry, $11 \%$ developed acute ischemic stroke. Old patients with COVID-19 were more prone to acute CVA[8].

With respect to our 70-year-old female patient infected with COVID-19, the deterioration in her clinical status with a baseline acute cardiac condition may have been due to a combination of lung injury and cardiac damage. We repeatedly performed echocardiography and detected RV dysfunction and exacerbated tricuspid regurgitation (by comparison with the previous echocardiography findings). Indeed the patient's dependence on inotropes made us suspicious of acute PTE. What further obfuscates the picture in patients with COVID-19 is the presence of tachypnea, tachycardia, hypoxia, and high D-dimer levels, rendering the differentiation between this infection and PTE challenging. High D-dimer is associated with a poor prognosis among patients with COVID-19 whether or not there is concomitant PTE.

We cannot be certain about the exact time of the occurrence of PTE in our patient. It is likely that PTE occurred in the hospital following PCI and her unwanted immobility due to hypoxia and femoral puncture. She was on therapeutic doses of heparin before PCI and prophylactic doses afterward. Another theory may be the coincidence of acute coronary syndrome, COVID-19, and PTE, which prompted the patient to refer to our emergency department, albeit with a 4-day delay.

Since COVID-19 patients are predisposed to thromboembolism, early diagnosis and treatment of such concomitant problems will improve their condition. We should be cautious to ascribe every sign and symptoms during hospitalization to just COVID-19.

Alongside coagulopathy, COVID-19 causes significant hypoxia along with an inflammatory milieu, which may lead to secondary myocardial infarction and also atherosclerotic plaque rupture. Hence, patients with COVID-19 are predisposed to both embolic and atherosclerotic events due to baseline inflammation[9].

\section{CONFLICT OF INTEREST}

The authors have no conflict of interest to declare.

\section{Ethical Approval Code: IR-TUMS.VCR.REC.1399.011}

\section{AUTHOR CONTRIBUTION}

$\mathrm{MN}$ and BH: gathered all clinical materials and images.YJ: performed coronary angiography. SS: Reported patients CT scan. KH: reviewed the literature and drafted the initial version of manuscript and performed echocardiography.NR: revised the manuscript.

\section{REFERENCES}

1. Liu, R., et al., Association of Cardiovascular Manifestations with In-hospital Outcomes in Patients with COVID-19: A Hospital Staff Data. medRxiv, 2020.

2. Sahin, A.R., et al., 2019 novel coronavirus (COVID-19) outbreak: a review of the current literature. EJMO, 2020.4 (1): p. 1-7.

3. Organization, W.H., Coronavirus disease 2019 (COVID-19): situation report, 67. 2020.

4. Bikdeli, B., et al., COVID-19 and thrombotic or thromboembolic disease: implications for prevention, antithrombotic therapy, and follow-up. Journal of the American College of Cardiology, 2020.

5. Thachil, J., et al., ISTH interim guidance on recognition and management of coagulopathy in COVID-19. Journal of Thrombosis and Haemostasis, 2020. 18 (5): p. 1023-1026. 
6. Poissy, J., et al., Pulmonary Embolism in COVID-19 Patients: Awareness of an Increased Prevalence. Circulation, 2020.

7. Oxley, T.J., et al., Large-Vessel Stroke as a Presenting Feature of Covid-19 in the Young. New England Journal of Medicine, 2020: p. e60.

8. Li, Y., et al., Acute cerebrovascular disease following COVID-19: a single center, retrospective, observational study. 2020.

9. Branchford, B.R. and S.L. Carpenter, The role of inflammation in venous thromboembolism. Frontiers in pediatrics, 2018. 6 : p. 142.

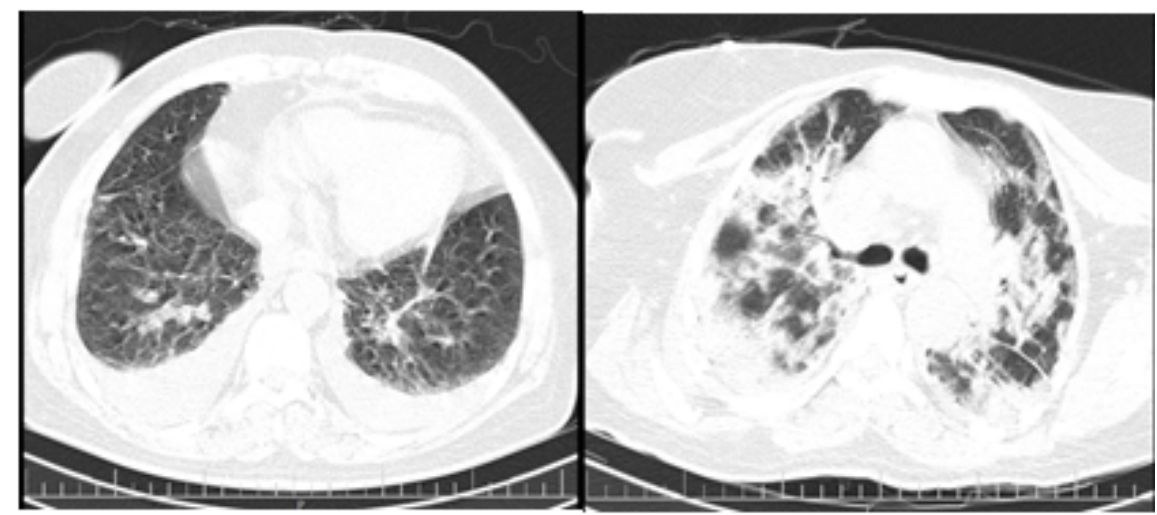

Figure 1. Left panel: First chest computed tomography scan. Right Panel: Second chest computed tomography scan 3 days later.

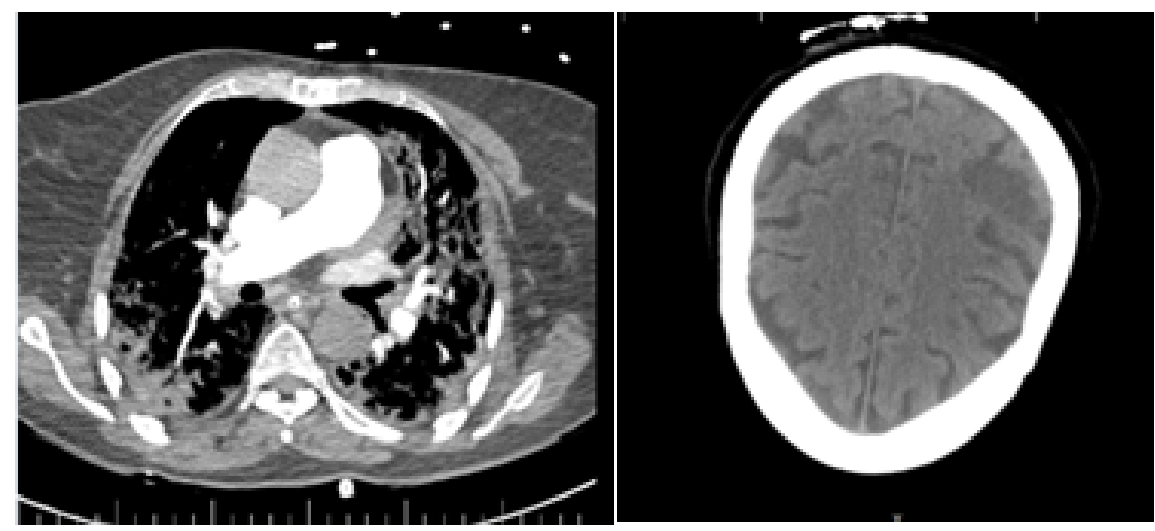

Figure 2. Left panel: Pulmonary thromboembolism. Right panel: Brain CT.

Hosted file

Figures.docx available at https://authorea.com/users/321827/articles/450994-occurrence-ofacute-coronary-syndrome-pulmonary-thromboembolism-and-cerebrovascular-event-in-covid-19 